\title{
Frequency of central lesions in polyneuropathy associated with IgM monoclonal gammopathy: an MRI, neurophysiological and immunochemical study
}

J M Léger, A Ben Younes-Chennoufi, M Zuber, P Bouche, M O Jauberteau, D Dormont, F Danon, N Baumann, P Brunet

Clinique des Maladies du Système Nerveux, Hôpital de la Salpêtrière, Paris

J M Lèger

M Zuber

$P$ Bouche

D Dormont

P Brunet

INSERM Unit 134,

Hôpital de la

Salpêtriêre, Paris,

France

A B Younes-Chennoufi

M O Jauberteau

N Baumann

Laboratoire

d'Immunologie. 11108.

Hôpital Saint-Louis,

France

F Danon

Correspondence to:

J M Léger, Clinique des

Maladies du Systeme

Nerveux, Hôpital de la

Salpêtrière, 47 boulevard de

l'Hôpital, 75651 Paris Cedex

13, France

Received 27 July 1990

and in revised form

Accepted 7 March 199

\begin{abstract}
CNS lesions were studied in polyneuropathy associated with IgM monoclonal gammopathy. Eleven out of 12 patients with IgM MGUS and one patient with Waldenstrom's disease had clinical and electrophysiological features indicating a demyelinating polyneuropathy. MRI showed CNS white matter lesions in two cases. Antibodies reacting against glycolipids present in CNS white matter were present in five cases, two of which had abnormal MRI. Central conduction times cortex-C7, obtained by magnetic stimulation, were prolonged in $3 / 8$ patients, of which two patients had anti CNS glycolipid antibodies.
\end{abstract}

Evidence for CNS demyelimination was recently suggested in chronic inflammatory demyelinating polyneuropathy (CIDP)..$^{1-3}$ In these studies, MRI revealed periventricular, subcortical and brainstem white matter lesions, indistinguishable from those seen in multiple sclerosis (MS), in patients with CIDP and either clinical evidence, or no signs of CNS involvement. In addition, prolonged central conduction times in some cases suggested CNS demyelination. ${ }^{12}$ Conversely, more recent MRI studies ${ }^{45}$ failed to find a significant frequency of typical MS-like lesions in patients with CIDP. Polyneuropathy associated with IgM monoclonal gammopathy of unknown significance (IgM MGUS) often presents with clinical and electrophysiological features similar to those of CIDP. ${ }^{6}$ The frequency of tremor and ataxia in these neuropathies, raised questions about an underlying CNS involvement. We aimed to discover if $\mathrm{CNS}$ demyelination could be found in IgM polyneuropathy.

\section{Patients and methods}

We studied 13 patients with a peripheral neuropathy associated with IgM monoclonal gammopathy. Laboratory investigations (except serum protein electrophoresis and immunoelectrophoresis), skeletal radiographs, abdominal CT and bone marrow histology were normal, indicating a monoclonal gammopathy of unknown significance (MGUS) in 12 out of 13 cases. Case 9 had Waldenstrom's disease.
Serum specimens were analysed for monoclonal immunoglobulin detection and antimyelin-associated-glycoprotein (anti-MAG) antibodies. CSF protein was available in 11 cases. Electrodiagnosis studies included examination with a concentric needle electrode, and nerve conduction velocities in all cases. Motor nerve conduction velocities (MNCV) were determined on peroneal and median nerves by surface electrodes. Criteria for demyelinating polyneuropathies were those defined by Kelly. ${ }^{8}$ Additionally the distal latency on the facial nerve was measured in 12 out of 13 patients.

1) MRI imaging

MRI examinations of the head were performed on a 0.5 Tesla MR unit (Magniscan 5000) with contiguous sagittal $T 1$ weighted slices 400 per 12 (TR/TE) and axial T2 weighted slices (1800 per 60,2 echos).

\section{2) Anti-glycolipid antibodies}

Antibodies directed against glycolipids purified from 1) human peripheral nerve system (PNS), and 2) CNS white matter were sought. ${ }^{9}{ }^{10}$ Briefly the acidic glycolipid fractions were obtained from human PNS and CNS after partition of the lipid extract; they contained mainly gangliosides, sulphatides (3-sulphategalactosyl-ceramide), and sulphated glucuronyl-glycolipids. Neuraminic acid content was measured according to Svennerholm. ${ }^{11}$ Immunodetection of the glycolipid antibodies ${ }^{1012}$ was performed after thin layer chromatographic (TLC) separation of the glycolipids. The position of these lipids on TLC was compared with known standards revealed by alpha-naphthol reagent specific for sugars. ${ }^{13}$ Briefly the sera of the patients were always used at a dilution of 1:100 (in phosphate-buffered saline solution containing $1 \%$ gelatin and $10 \%$ inactivated horse serum) and incubated for two hours at $37^{\circ} \mathrm{C}$. The antigenantibody complex was developed using a peroxidase-labelled second antibody, which was a goat anti-human IgM specific for mu chain (Cappel, USA), and 4-chloro-1-naphthol reagent to visualise the peroxidase reaction product. $^{12}$

\section{3) Central conduction times}

Transcranial stimulation of the frontal cortex and a percutaneous stimulation at the $\mathrm{C} 7$ spinal cord level were performed in eight out of 13 cases using a Novametrix magnetic coil 
Table 1 Clinical and laboratory data

\begin{tabular}{|c|c|c|c|c|c|c|c|c|c|c|c|}
\hline \multirow[b]{2}{*}{$\begin{array}{l}\text { No of } \\
\text { cases }\end{array}$} & \multirow[b]{2}{*}{ Age } & \multirow[b]{2}{*}{$\begin{array}{l}\text { Sex } \\
\text { ratio }\end{array}$} & \multirow[b]{2}{*}{$\begin{array}{l}\text { Evolution } \\
\text { (years) }\end{array}$} & \multirow[b]{2}{*}{$\begin{array}{l}\text { Motor } \\
\text { weakness }\end{array}$} & \multirow[b]{2}{*}{ Ataxia } & \multirow[b]{2}{*}{ Tremor } & \multirow[b]{2}{*}{$\begin{array}{l}\begin{array}{l}\text { Protein } \\
(\mathrm{g} / \mathrm{l})\end{array} \\
\end{array}$} & \multicolumn{2}{|c|}{ Gammopathy } & \multirow[b]{2}{*}{ Anti-MAG } & \multirow[b]{2}{*}{ Treatments } \\
\hline & & & & & & & & $\operatorname{Ig} M(g / l)$ & Light chain & & \\
\hline 1 & 74 & $\mathbf{M}$ & 3 & & + & + & 0.98 & 7 & $\kappa$ & + & $I S+P$ \\
\hline 2 & 69 & $\mathbf{M}$ & 2 & & & & 0.93 & 6 & $\hat{\kappa}$ & + & \\
\hline 3 & 57 & $\mathbf{M}$ & 5 & + & + & + & 1.44 & $5 \cdot 7$ & $\hat{\lambda}$ & + & IS \\
\hline $\begin{array}{l}3 \\
4\end{array}$ & 55 & $M$ & 8 & + & + & + & 1.27 & 3 & $\hat{\lambda}$ & $T$ & \\
\hline 5 & 53 & $\mathbf{M}$ & $4 \cdot 5$ & & + & & 0.55 & 3 & $\lambda$ & & $C+P$ \\
\hline 6 & 78 & $\mathbf{F}$ & 4 & & + & + & 0.59 & 6 & $\kappa$ & + & \\
\hline 7 & 84 & $\mathbf{F}$ & 2 & + & + & & 0.56 & 11 & $\lambda$ & & IS $=P$ \\
\hline 8 & 70 & $\mathbf{M}$ & 4 & & + & + & 0.75 & 4 & $\kappa$ & + & \\
\hline 9 & 60 & $\mathbf{F}$ & 6.5 & + & + & & 0.62 & 6 & $\kappa$ & + & IS $+\mathbf{P}$ \\
\hline 10 & 46 & $\mathbf{F}$ & 2 & & & & & $4 \cdot 7$ & $\kappa$ & + & \\
\hline 11 & 51 & $\mathbf{F}$ & 11 & + & + & & 0.77 & $14 \cdot 8$ & $\kappa$ & + & IS $+\mathbf{P}$ \\
\hline 12 & 61 & $\mathbf{M}$ & 5 & & & & & 8 & $\kappa$ & + & IS \\
\hline 13 & 48 & $\mathbf{M}$ & 8 & + & + & + & 1.52 & $7 \cdot 3$ & $\kappa$ & + & IS \\
\hline
\end{tabular}

$\mathbf{M}=$ Male

$\mathbf{F}=$ Female

$+=$ Presence of

IS = Immunosuppressive treatment by chlorambucil

$\mathrm{C}=$ Corticosteroid treatment

$\mathrm{P}=$ Plasmaphereses

$\mathbf{O}=$ No treatment

stimulator, according to the technique initially reported by Barker et al. ${ }^{14}$ Muscle action potentials (MAP) were recorded from the abductor digiti minimi. The central conduction times were obtained by calculating the latency difference between cortex and cord responses, and compared with those of 15 control subjects from our laboratory.

\section{Results}

Clinical and laboratory data are reported in Table 1. All patients complained of paresthesiae of the lower extremities, and a distal sensory loss, affecting mainly vibration and position sensation, was found in the feet and in four cases in the hands. Ataxia was found in 10 cases, and there was a postural tremor of the upper limbs in five cases. No other clinical sign of involvement of the CNS was found, and there was no sign suggesting a hyperviscosity syndrome. The monoclonal IgM globulin concentrations at the time of the study ranged from 3-15.8 g/l. In patient 9 with Waldenstrom's disease, the paraprotein concentration at the time of the diagnosis (three years before) was $28 \mathrm{~g} / \mathrm{l}$. Anti-MAG activity was demonstrated in 10 cases (cases 1, 2, 3, 6, 8-13); eight of which (cases $1,3,6,8,10-13$ ) also expressed reactivity against the sulphated glucuronyl paragloboside (SGPG), which has been commonly found in

Table 2 Electrophysiological data

\begin{tabular}{llclcc}
\hline $\begin{array}{l}\text { No of } \\
\text { cases }\end{array}$ & $\begin{array}{l}\text { Median } M C V \\
(\mathrm{~m} / \mathrm{s})\end{array}$ & $\begin{array}{l}D L \\
(\mathrm{~ms})\end{array}$ & $\begin{array}{l}\text { Peroneal } M C V \\
(\mathrm{~m} / \mathrm{s})\end{array}$ & $\begin{array}{l}\mathrm{DL} \\
(\mathrm{ms})\end{array}$ & $\begin{array}{l}\text { Facial nerve } D L \\
(\mathrm{~ms})\end{array}$ \\
\hline 1 & 40 & $9 \cdot 4$ & 26 & $16 \cdot 5$ & $3 \cdot 3$ \\
2 & 36 & 16 & 22 & $10 \cdot 4$ & $3 \cdot 1$ \\
3 & 41 & $8 \cdot 1$ & 31 & $15 \cdot 3$ & $3 \cdot 3$ \\
4 & 56 & $4 \cdot 1$ & 40 & 8 & $3 \cdot 4$ \\
5 & 47 & $4 \cdot 3$ & 28 & $7 \cdot 7$ & $3 \cdot 7$ \\
6 & 36 & $7 \cdot 8$ & 17 & $12 \cdot 3$ & $10 \cdot 1$ \\
7 & 48 & $3 \cdot 6$ & 40 & $4 \cdot 3$ & $2 \cdot 6$ \\
8 & 19 & $7 \cdot 2$ & NO & & $4 \cdot 7$ \\
9 & NO & $8 \cdot 8$ & NO & & $5 \cdot 2$ \\
10 & 48 & $6 \cdot 4$ & 39 & $8 \cdot 8$ & ND \\
11 & 16 & $19 \cdot 1$ & NO & & $5 \cdot 1$ \\
12 & 45 & $6 \cdot 3$ & 20 & 19 & $4 \cdot 1$ \\
13 & 27 & $9 \cdot 8$ & NO & $9 \cdot 5$ & $6 \cdot 2$ \\
\hline
\end{tabular}

$\mathrm{MCV}=$ Motor conduction nerve velocities

$\mathrm{DL}=$ Distal latencies

NO $=$ Not obtained

$\mathrm{ND}=$ Not done this neuropathy. ${ }^{915}$ Nine out of 13 patients received immunosuppressive treatments (corticosteroids, plasmaphereses, chlorambucil) which were sometimes followed by transient improvement of the sensory symptoms and/or by a diminution of the monoclonal IgM globulin concentration (as in case 9). However, the clinical and the electrophysiological signs of the neuropathy remained unchanged or slightly worsened.

Electrophysiological data are summarised in Table 2. Motor nerve conduction velocities were considerably reduced and/or motor distal latencies were prolonged in at least two peripheral nerves in 11 out of 13 cases, suggesting a demyelinating pattern according to Kelly's criteria. ${ }^{8}$ No conduction block was found. Motor nerve conduction velocities were not obtained in case 9 because of a severe distal muscle atrophy. Only in case 7, were electrophysiological data consistent with an axonopathy. In addition the distal latency on the facial nerve was prolonged in four of 12 cases ( $>5 \mathrm{~ms}$ ). Nerve biopsies were abnormal in nine of 13 cases in which they were obtained, and showed a chronic demyelinating polyneuropathy with extensive accompanying axonal loss. Teased nerve fibres were available in cases 5 and 10 and showed respectively: case $5=$ normal fibres $29 \cdot 8 \%$, segmental demyelination $17 \%$, axonal degeneration $53.2 \%$; case $10=$ normal fibres $64 \%$, segmental demyelination $15 \%$, axonal degeneration $21 \%$.

MRI was abnormal in two cases. In case 8, a 70 year old man, widespread thin periventricular lesions and other small, high signal lesions in the subcortical white matter and in the right cerebellum hemisphere, were seen on T2 weighted slices (fig 1). In case 11 , a 51 year old women, MRI showed small, high-signal lesions in the subcortical white matter on both sides, predominant in the occipital lobes (fig 2).

Antibodies reacting against glycolipids present in the CNS white matter were found in five cases (cases $1,3,4,8,11$ ), as illustrated in fig 3 (case 3 ). In these five cases, the IgM reacted mainly in the PNS and CNS with a lipid migrating on thin-layer chromatography at the sulphatide level. This lipid was not a sulphatide but remains as yet uncharacterised. ${ }^{10}$ In four of 
Figure 1 MRI (T2 weighted slices of the head): Case 8: Widespread thin periventricular high signal.

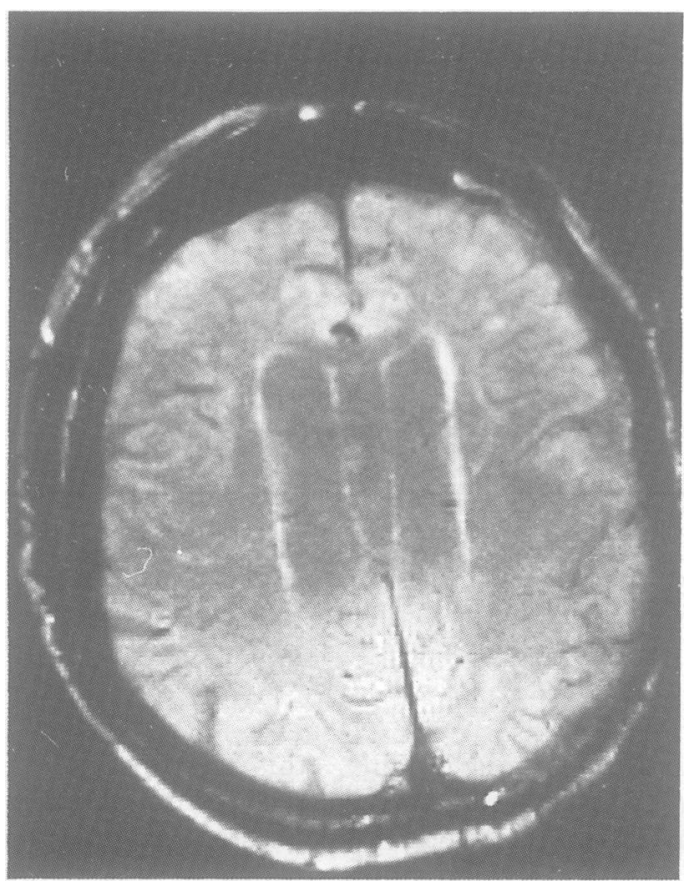

these five cases (cases $1,4,8,11$ ) the IgM did not react with the purified sulphatide standard; in one case (case 3 ), there was also a slight reactivity towards the sulphatide standard.

The central conduction times cortex-C7 were prolonged in 3 out of 8 patients: cases 3 , 4 and 5 (respectively: $8.8,8.2,10.5 \mathrm{~ms}$ ), if compared to our control series (mean value: $7 \cdot 1 \pm 1 \cdot 2 \mathrm{~ms}$. Upper limit: $8 \cdot 1 \mathrm{~ms}$ ).

Two of the 5 cases with anti-CNS glycolipid antibodies (cases 8 and 11) had MRI abnormalities. Two other cases with anti-CNS glycolipid antibodies (cases 3 and 4) had prolonged central conduction times. There was no direct correlation between the cases with MRI abnormalities and the cases with prolonged central conduction times.

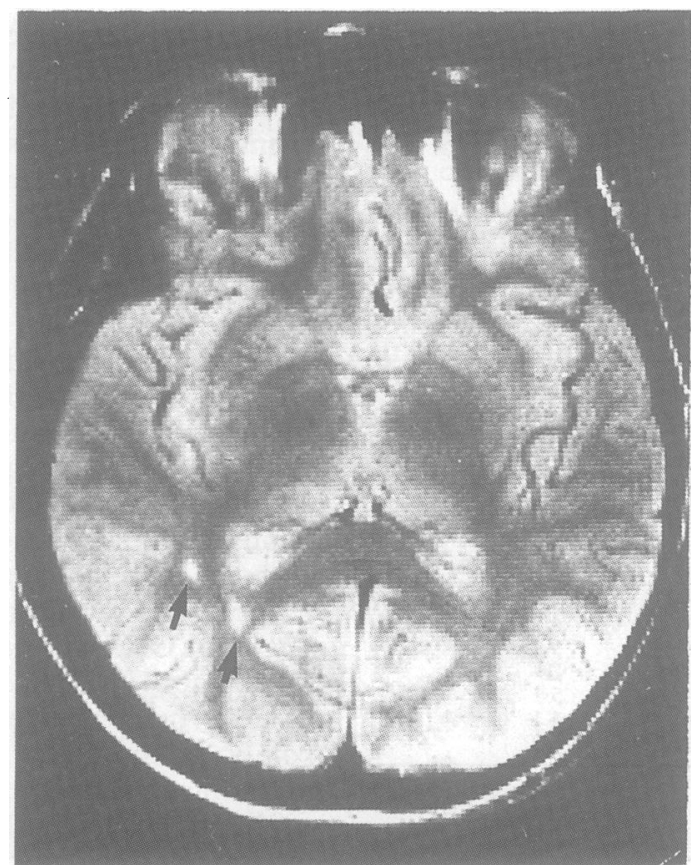

\section{Discussion}

Polyneuropathy associated with IgM MGUS is now a well-recognised syndrome. ${ }^{71016}$ The electrophysiological findings usually indicate a demyelinating process ${ }^{817}$ which seems to affect the entire length of all motor nerve fibres. ${ }^{16}$ IgM M-protein is shown to bind frequently both to myelin-associated-glycoprotein (MAG) and SGPG. ${ }^{915}$ In our series, 11 out of 13 cases with IgM MGUS and case 9 with Waldenstrom's disease fulfilled clinical and electrophysiological criteria for demyelinating polyneuropathy, according to Kelly's findings in MAG-reactive patients. ${ }^{8}$ The absence of conduction blocks rather indicated a diffuse peripheral demyelination. The increase of the distal latency of the facial nerve in 1 out of 3 of these cases may indicate an asymptomatic involvement of this cranial nerve. The pathological findings in our nerve biopsies were similar to those of the literature, showing a mixed pattern of demyelination and axonal loss. ${ }^{16}$ Eight of these 12 cases were MAG + and SGPG +, and 2 cases were MAG - and SGPG - , demonstrating a good correlation between these two antigens which have a common epitope. Cases 2 and 9 were MAG + and SGPG - , indicating that their IgM did not recognise exactly the same epitope. On the other hand, an axonopathy, as observed in case 7, with lack of anti-MAG and anti-SGPG antibodies, was rarely reported in IgM polyneuropathy ${ }^{18}$ : in our case, the neuropathy was not different from the majority group; concerning laboratory data and follow up. Chlorambucil and plasma exchanges ${ }^{19}$ have been shown to be effective in some neuropathies associated with monoclonal IgM. The results of the treatment by chlorambucil and plasma exchanges in our cases did not differ from those

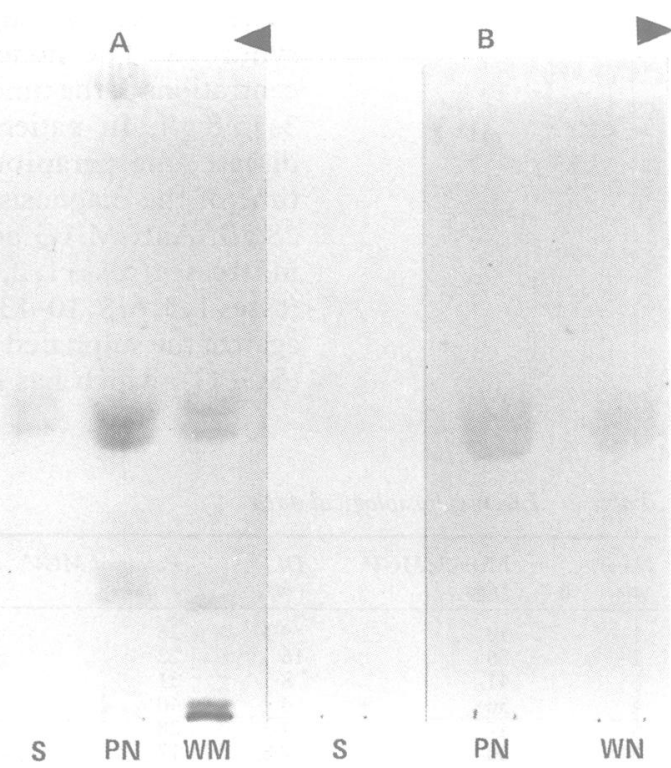

Figure 3 Binding of IgM from patient 3 to human CNS and PNS glycolipids separated on thin-layer

chromatography. A: Staining of glycolipids with alphanaphtol reagent. $B$. Immunodetection of glycolipids using MGUS serum $1: 100$ and peroxydase-labelled anti IgM (mu chain) second antibody. S: sulphatide standard $(8 \mu g)$. PN: peripheral nerve glycolipids (corresponding to 10 ug neuraminic acid). WM: CNS white matter glycolipids (corresponding to 20 um neuraminic acid). 
previously reported, and from the preliminary results of the French cooperative study (to be published).

Questions persist regarding ataxia and tremor of the upper limbs in these patients. It has been suggested that they may be related to a disturbance of peripheral sensation, to slowing of nerve conduction in the servomotor loop, or else to an associated disorder of the central nervous system. ${ }^{20}$ Smith et al ${ }^{16}$ noticed that the severity of the tremor and ataxia was not correlated with the degree of sensory loss, nor with the slowing of nerve conduction. They suggested that the abnormal movements had some of the characteristics of central disorders such as Parkinsonism or cerebellar disease, and were often similar to the abnormalities found in benign essential tremor. On the other hand, it is known that the most prevalent antibody activity among the IgM polyneuropathies is directed against MAG, which is found in both peripheral and central myelin. ${ }^{21}$ However, the pathogenic role of anti-MAG antibodies needs still to be demonstrated. ${ }^{22}$

In our series, gait ataxia was frequent, and postural, essential-like, tremor of the upper limbs was present in nearly half of the cases, but ataxia and tremor were not clearly correlated with the severity of the peripheral symptoms and signs, nor with the abnormalities of the electrophysiological study. Although no patient had other symptoms suggesting a CNS involvement, it was looked for systemically. The abnormal MRI white matter signals in two cases suggested an involvement of the CNS white matter. Their frequency (2 out of 13) was not significant if compared to those of elderly patients' control series in the literature. ${ }^{23}$ Abnormal white matter signals in patients with CIDP are more frequent in cases older than $50 .^{25}$ Our 2 patients with MRI lesions were older than 50 , but they fulfilled criteria 1 and 2 suggested for primary demyelination in elderly patients ${ }^{24}$ (two or more of the following findings (a) lesion size $>6 \mathrm{~mm}$, (b) lesions abutting the bodies of the lateral ventricles, and (c) infratentorial white matter lesions. These data are different from MRI findings in 5 cases of chronic demyelinating polyneuropathy associated with benign monoclonal paraproteinaemia ( 2 IgG and 3 IgM) reported by Hawke et $a l^{5}$ normal or considered as non-specific.

In addition the demonstration of antibodies reacting with glycolipids present in the CNS white matter in our 2 cases with abnormal MRI (and three other cases) may favour the hypothesis of a CNS demyelination. Furthermore, no anti-CNS glycolipid antibodies were found in 30 control patients of the same age groups, without neuropathy and with (10 cases) or without (20 cases) IgM gammopathy (Ben Younes-Chennoufi (in press). Three out of 8 cases which performed magnetic stimulation, also had prolonged central conduction times $(2$ of which had anti-CNS glycolipid antibodies), which may also indicate a CNS demyelination.

In the 5 patients who had anti-CNS glycolipid antibodies (cases 1, 3, 4, 8 and 11), there was a good clinical correlation for ataxia (present in all cases), and a moderate one for the tremor of the upper limbs ( 3 out of 5 cases).
MRI will be repeated in the 3 cases with presence of anti-CNS glycolipid antibodies but normal MRI, to determine whether the presence of anti-CNS glycolipid antibodies is a significant early indication of CNS involvement.

We thank Dr M C Diemer for her contribution to this study and Dr H Ratinahirana for performing the histological study. The sulphatide standard was donated by Professor R K Yu, Virginia Commonwealth University. Brain ganglioside standard mixture was donated by Fidia (Abano Terme).

1 Thomas PK, Walker RWH, Rudge P, et al. Chronic demyelinating peripheral neuropathy associated with multifocal central nervous system demyelination. Brain 1987;110:53-76.

2 Ormerod IEC, Waddy HM, Kermode AG, Murray NMF, Ormerod IEC, Waddy HM, Kermode AG, Murray NMF,
Thomas PK. Involvement of the central nervous system in chromic inflammatory demyelinating polyneuropathy: a clinical, electrophysiological and magnetic resonance imaging study. J Neurol Neurosurg Psychiatry 1990; 53:789-93.

3 Mendell JR, Kolkin S, Kissel JT, et al. Evidence for central nervous system demyelination in chronic inflammatory demyelinating polyneuropathy. Neurology 1987;37: 1291-4.

4 Feasby TE, Hahn AF, Koopman WJ, Lee DH. Central lesions in chronic inflammatory demyelinating polyneuropathy: an MRI study. Neurology 1990;40:476-8.

5 Hawke SHB, Hallinan JM, McLeod JG. Cranial magnetic resonance imaging in chronic demyelinating poly-
neuropathy. J Neurol Neurosurg Psychiatry 1990;53: 794-6.

6 Dyck PJ. Intravenous immunoglobulin in chronic inflammatory demyelinating polyneuropathy and in neuropathy associated with IgM monoclonal gammopathy of unknown significance. Neurology 1990;40:327-8.

7 Léger JM, Zuber M, Chennoufi A, et al. Central nervous system demyelination in polyneuropathy associated with benign IgM monoclonal gammopathy. Neurology 1989;39 (Suppl 1):393-4.

8 Kelly JJ. The electrodiagnostic findings in polyneuropathies associated with IgM monoclonal gammopathies. Muscle and Nerve 1990;13:1113-7.

9 Jauberteau MO, Hénin D, Bouche P, et al. Etude des anticorps antiglycolipides au cours des dysglobulinémies monoclonales à IgM associées à une neuropathie périphéque. Rev Neurol (Paris) 1988;144:474-80.

10 Jauberteau MO, Ben Younes-Chennoufi A, Rigaud M, Baumann N. IgM gammopathy and polyneuropathy react
with an antigenic glycolipid present in human central with an antigenic glycolipid present in hum
nervous system. Neurosci Lett 1989;97:181-4.

11 Svennerholm L. Quantitative estimation of sialic acids. II. A colorimetric resorcinol-hydrochloric method. Biochim Biophys Acta 1957;24:604-11.

12 Mailly P, Ben Younes-Chennoufi A, Bon S. The monoclonal antibodies Elec-39, HNK-1 and NC-1 recognize common structures in the nervous system and muscles of vertebrates. Neurochem Int 1989;15:517-30.

13 Jacin H, Mishkin AR. Separation of carbohydrates on borate-impregnated silica gel $G$ plates. J Chromatogr 1965;18:170-3.

14 Barker AT, Freeston IL, Jalinous R, Jarrat JA. Noninvasive magnetic stimulation of the human motor cortex. Lancet 1985;2:1106-7.

15 Chou KH, Ilyas AA, Evans JE, et al. Structure of sulfated glucuronyl glycolipids in the nervous system reacting with glucuronyl glycolipids in the nervous system reacting with HNK-1 antibody and some IgM parap
pathy. $J$ Biol Chem 1986;261:11717-25.

16 Smith IS, Kahn SN, Lacey BW, et al. Chronic demyelinating neuropathy associated with benign IgM paraproteinemia. Brain 1983;106:169-95.

17 Bouche P, Bouchacourt E, Léger JM, Travers MA, Cathala HP. Les neuropathies périphériques associées aux gammapathies monoclonales. Intérêt des études électrophysiologiques. Rev EEG Neurophysiol 1985;15:255-65.

18 Sherman WH, Latov N, Hays AP, et al. Monoclonal IgM K antibody precipitating with chondroitin sulfate $C$ from patients with axonal polyneuropathy and epidermolysis. Neurology 1983;33:192-201.

19 Lassoued K, Dellagi K, Brouet JC, et al. Effects of plasma exchange in 9 patients with peripheral neuropathy and monoclonal IgM directed to myelin-associated glyco-

20 Adams RD, Shahani BT, Young RR. Tremor in association with polyneuropathy. Trans Am Neurol Assoc 1972;97: with 8 .

21 Braun PE, Frail DE, Latov N. Myelin associated glycoprotein is the antigen for a monoclonal IgM in poly-

22 Mendell JR, Sahenk Z, Whitaker JN, et al. Polyneuropathy and IgM monoclonal gammopathy: studies on the pathogenic role of anti-myelin-associated glycoprotein antibody. Ann Neurol 1985;17:243-54.

23 Hunt AL, Orrison WW, Yeo RA, et al. Clinical significance of MRI white matter lesions in the elderly. Neurology 1989;39:1470-4.

24 Fazekas F, Offenbacher $H$, Fuchs $S$, et al. Criteria for an increased specificity of MRI interpretation in elderly subjects with suspected multiple sclerosis. Neurology 1988;38:1822-5. 